\title{
The split in the ancient cold front in the Perseus cluster
}

Stephen A. Walker ${ }^{1 *}$, John ZuHone ${ }^{2}$, Andy Fabian ${ }^{3}$, Jeremy Sanders ${ }^{4}$

${ }^{1}$ Astrophysics Science Division, X-ray Astrophysics Laboratory, Code 662, NASA Goddard Space Flight Center, Greenbelt, MD 20771, USA

${ }^{2}$ Harvard-Smithsonian Center for Astrophysics, 60 Garden St., Cambridge, MA 02138, USA

${ }^{3}$ Institute of Astronomy, Madingley Road, Cambridge CB3 OHA

${ }^{4}$ Max-Planck-Institute fur extraterrestrische Physik, 85748 Garching, Germany

Sloshing cold fronts in clusters, produced as the dense cluster core moves around in the cluster potential in response to in-falling subgroups, provide a powerful probe of the physics of the intracluster medium $(I C M)$, and the magnetic fields permeating $\mathrm{it}^{1 / 1 / 2}$. These sharp discontinuities in density and temperature rise gradually outwards with age in a characteristic spiral pattern, embedding into the intracluster medium a record of the minor merging activity of clusters: the further from the cluster centre a cold front is, the older it is. Recently it has been discovered that these cold fronts can survive out to extremely large radii in the Perseus cluster ${ }^{3}$. Here we report on high spatial resolution Chandra observations of the large scale cold front in Perseus. We find that rather than broadening through diffusion, the cold front remains extremely sharp (consistent with abrupt jumps in density) but instead is split into two sharp edges. These results show that magnetic draping can suppress diffusion for vast periods of time, around $\sim 5 \mathrm{Gyr}$, even as the cold front expands out to nearly half the cluster virial radius.

One of Chandra's first advances in understanding galaxy clusters was the discovery of cold fronts: sharp edges in X-ray surface brightness where the gas density rapidly drops while the temperature rises, the opposite of a shock ${ }^{4 / 5}$. The exceptional sharpness of these cold fronts, smaller than the Coulomb mean free path, indicates that diffusion and conduction processes in the ICM (which should smooth the edges in density and temperature) and hydrodynamic instabilities are suppressed, most likely due to magnetic draping 6 (9). Detailed studies of sloshing cold fronts have at present been limited to those in the central $\sim 100 \mathrm{kpc}$, where the ICM is brightest and the cold fronts are young.

One remarkable recent development is the discovery of large scale cold fronts reaching out to very large radii, around half the virial radius $(730 \mathrm{kpc})$ in Perseus ${ }^{33}$ and several other clusters ${ }^{10 \mid 11}$. These colossal cold fronts are the oldest coherent structures surviving in cool core clusters, with the one we present here in Perseus being around $\sim 5$ Gyr old (see Figs 1, 2, and supplementary figure 3). By comparison the structures seen in cluster cores produced by AGN feedback are being constantly dissipated and replaced on timescales of just $\sim 100$ s Myr.

As cold fronts rise outwards the resulting velocity shear should lead to Kelvin-Helmholtz

*Email: stephen.a.walker@nasa.gov 
instabilities $(\mathrm{KHI})^{12}+14$, as we have found in younger cold fronts ${ }^{15}$. Magnetic draping over the cold front surfaces has been found to inhibit $\mathrm{KHI}^{15 / 16}$. In ancient cold fronts, much more time has elapsed over which KHI can develop, and over which diffusion and conduction processes can smooth over the jumps in density and temperature, providing powerful tests of simulations ${ }^{917}$.

These ancient cold fronts have risen slowly outwards from the core, experiencing vastly different ICM environments. In cluster cores AGN feedback dominates ${ }^{18}$, with sound waves ${ }^{19}$ and turbulent gas motions ${ }^{20}$ likely dissipating the feedback energy into the ICM. They then move away from the core, where AGN feedback no longer dominates. Moving into the cluster outskirts, the turbulence and gas motions in the ICM should rise again21, due to the continuing accretion of matter. By studying Chandra observations of the ancient cold front in Perseus, we can see the time integrated effect of all of these processes on the cold front.

The existing shallow XMM mosaic of Perseus is shown in Fig. 1 (top). Overlaid on this is the gradient map from Gaussian Gradient Magnitude filtering ${ }^{2223}$ of the inner Chandra and outer XMM mosaics, emphasising the edges. The large cold front $730 \mathrm{kpc}$ to the east of the core is clear. From these wide-field data we obtain a large scale, low spatial resolution temperature map (Fig. 1, bottom left), showing the characteristic cold arch of gas behind the surface brightness edge. This is consistent with the expected temperature behaviour seen in simulations ${ }^{917}$, (Fig. 1, bottom right), for sloshing perpendicular to the line of sight. 

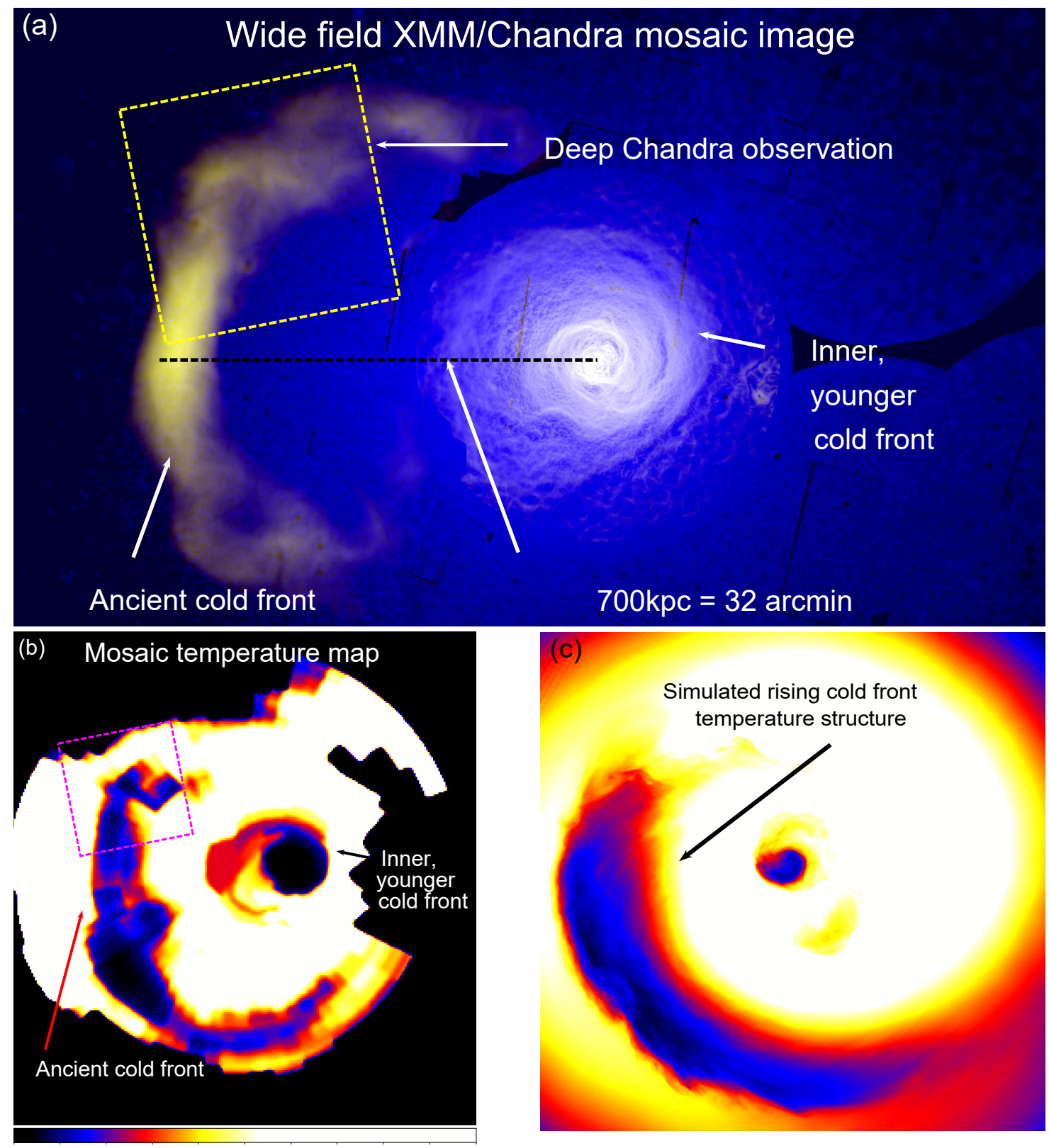

$\begin{array}{lllllllllll}2 & 3.5 & 3.7 & 3.8 & 3.9 & 4 & 4.2 & 4.3 & 4.5 & 4.7 & 7\end{array}$

Figure 1 Wide-field X-ray observations compared to simulations. Top: XMM-Newton mosaic of the Perseus cluster (blue), over which is laid the gradient map (white, pink and yellow) obtained from GGM filtering of the Chandra (inner) and XMM (outer) mosaics. The ancient, $730 \mathrm{kpc}$ radius outer cold front is clear on the left. The location of our new deep Chandra observations (presented in Fig. 2) is shown by the dashed box. Bottom left: Low spatial resolution large scale temperature map from the shallow wide-field XMM and Chandra mosaics, which shows the large cold arch of gas behind the ancient cold front. Bottom right: Temperature map from gas sloshing simulation ${ }^{17}$. The arch shaped cold band matches the geometry of the observed cold front arch seen in Perseus in the bottom left panel. 
Our new, high spatial resolution Chandra ACIS-I observations (Fig 2, left) have targeted the region to the north of the large scale cold front, (Fig. 1, dashed square). These show that, instead of the typical single cold front edge in the X-ray surface brightness, the cold front has split into 2 edges, separated by $\sim 100 \mathrm{kpc}$. The temperature map (Fig 2, right) shows that there are two cold arcs behind each X-ray surface brightness edge, separated by a band of hotter gas. Such cold front splitting has never been seen before.

The two surface brightness edges are remarkably sharp. When the surface brightness edges are fit with two broken powerlaws smoothed by a Gaussian (Supplementary figure 1), their widths are consistent with being zero. The conservative $(3 \sigma)$ upper limit on the width of both edges is 8 arcseconds $(3.1 \mathrm{kpc})$. This is much smaller than the Coulomb mean free path for diffusion from the inner (brighter) side to the outer side of $\lambda_{\text {in } \rightarrow \text { out }, 2}=23.2 \pm 6 \mathrm{kpc}(60.0 \pm 15 \mathrm{arcsec})$ for edge 2 , and $\lambda_{\text {in } \rightarrow \text { out }, 1}=9.6 \pm 1.5 \mathrm{kpc}(25 \pm 4 \mathrm{arcsec})$ for edge 1 . This shows that magnetic fields are still able to support the edges against broadening even at these extreme radii, and for huge periods of time.

Comparing our observed structures to simulations of sloshing with different strength initial cluster magnetic fields ${ }^{9}[17$ we find that one possibility for this 'double edge' cold front splitting in this region may be due to the development of features similar to the onset of a Rayleigh-Taylor (RT) instability (Fig. 3 and 4). In an RT instability, dense, low-entropy gas is suspended "above" (in the sense of the direction of the local gravitational acceleration) less dense, higher-entropy gas. This suspension is unstable, and produces the familiar RT "fingers" of dense gas falling into the lower-density material.

In these simulations, the cooler gas is initially displaced from the dark matter dominated potential by the "ram-pressure slingshot" $\operatorname{effect}^{24}$ (Fig 3, top left). As the gas falls back into the potential and overshoots it, it forms the first cold front. The lowest-entropy gas behind this front falls back into the cluster potential minimum (Fig. 3, top right) from a location slightly offset from the center of the front (similar to the RT "fingers"), while the rest of the front continues to propagate radially outward. The result is that the coldest, lowest-entropy part of the sloshing structure develops a "bend' from the initial front going counterclockwise inward toward the cluster center, but protruding outward from the edge of this bend is a "hook" feature which is a continuation of the curvature of the original front (Fig. 3, bottom). These simulations begin with an initial uniform thermal to magnetic pressure ratio $\left(\beta=p_{\mathrm{th}} / p_{\mathrm{B}}\right)$ throughout the cluster before any sloshing begins. As the sloshing proceeds, the magnetic field becomes amplified and wrapped around the cold fronts, affecting the development of the "hook" feature as we show later in Fig. 4. In our best matching simulation, the magnetic field draped along the cold front is typically 10 times stronger than the field outside the cold front, and is $\sim 5 \mu \mathrm{G}$ in the oldest simulation time slice. 


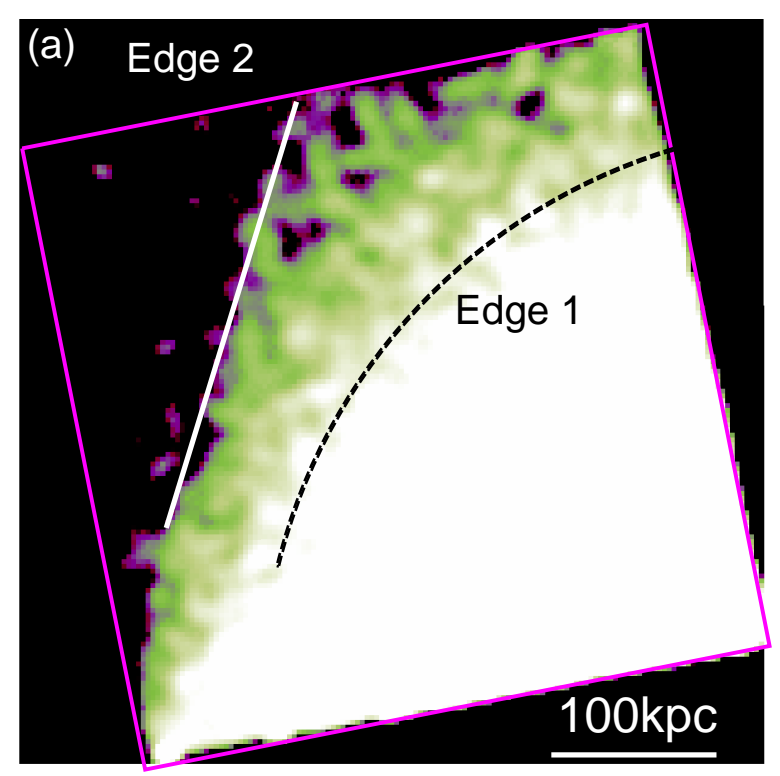

Deep Chandra observation

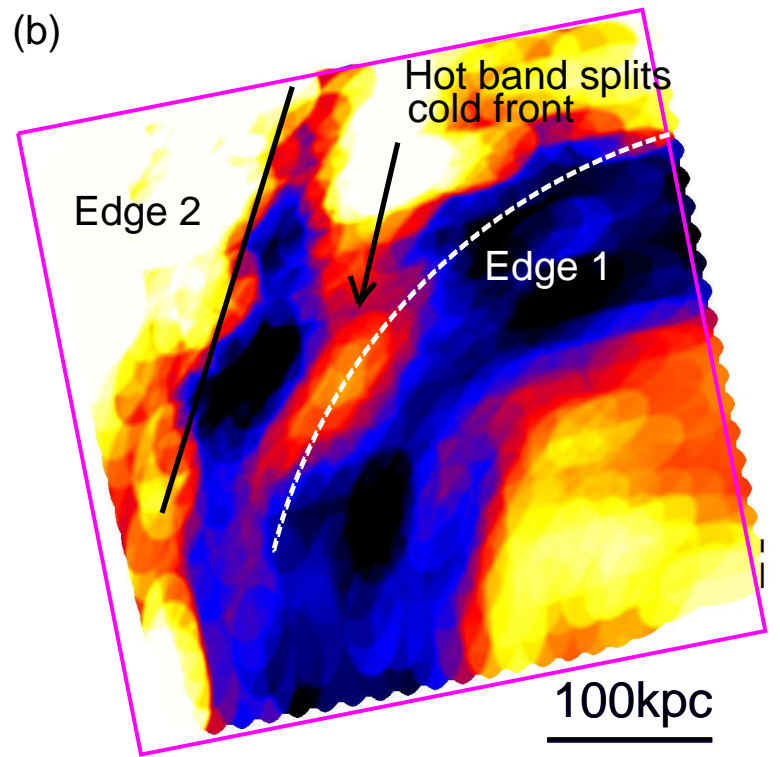

Chandra kT map

3.5

4

4.5

5

5.56

6.5

Figure 2 Deep Chandra observations. The left panel shows an image in the 1.24.0keV band from our $95 \mathrm{ks}$ Chandra observation, which maximises the contrast of the edges. Two edges are visible in the deep observation: 'edge 1' follows the curvature of the cold front shown in Fig 1, while the outer 'edge 2' is approximately straight. The right hand panel shows the temperature map, showing a sharp temperature jump at each edge, and a hot band splitting the cold front. The colorbar shows the temperature in keV.

This hook feature rises outwards with the cold front, and survives out to the final epoch of the simulation, at which the cold front is $3.3 \mathrm{Gyr}$ old (we define the age as the time since closest approach of the interacting clusters) and has risen to a radius of 450kpc (supplementary figure 4). Plotting the cold front radius against age from the simulation (supplementary figure 3), the relation is perfectly linear (a constant rise speed, as has been found in earlier works ${ }^{13}$ ). Since the thermodynamic and mass profiles of the cluster continue as simple powerlaws in the simulation from 100kpc outwards, it is possible that the hook can survive out to the observed cold front radius of $730 \mathrm{kpc}$, with the cold front continuing to rise with constant speed. Extrapolating the linear best fit, the Perseus cold front at $730 \mathrm{kpc}$ would be $5.2 \mathrm{Gyr}$ old, which we conservatively quote throughout as $\sim 5 \mathrm{Gyr}$.

This hook feature leads to two edges forming, separated by a hotter band, which continues to move outwards as the cold front rises, similar to what we observe (Fig. 4, middle column for the $\beta=200$ simulation). When the magnetic field is stronger (initial $\beta=100$ ), this splitting is suppressed, and the hot band separating the two edges does not form (Fig. 4, left column). When the magnetic field is weaker (initial $\beta=500$ ), the magnetic field is too weak to stop the cold front from breaking 
up into KHI, so the split cold front structure does not form (Fig. 4, right column).

While the simulations we use here only extend to a cold front age of $3.3 \mathrm{Gyr}$, previous simulations ${ }^{12 \mid 24}$ have successfully produced cold fronts out to $4.7 \mathrm{Gyr}$ from the time of closest approach, comparable to the $\sim 5 \mathrm{Gyr}$ estimated here for Perseus. We note that our simulations do assume the rigid potential approximation ${ }^{25}$, whose validity is unclear outside $500 \mathrm{kpc}$, (though simulations which do not use the rigid potential approximation ${ }^{24}$, also show the hook feature persisting to large radii and later epochs, so its presence and maintenance is not an artifact of this approximation). We stress that we are proposing a general explanation for the cold front splitting we observe, and attempting to reproduce it exactly in simulations is beyond the scope of this paper.

The double edge feature we observe may also be compatible with multiple edges seen in simulations of the Virgo cluster cold front for low $(0.1 \%$ Spitzer $)$ viscosity ${ }^{14}$ due to $\mathrm{KH}$ rolls, though at present these have only been simulated on much smaller scales ( $\sim 10 \mathrm{~s} \mathrm{kpc})$. The splitting may be compatible with a range of Reynolds numbers as well, but determining this is beyond the scope of this work.

The remarkable sharpness of the cold front edges shows that the magnetic field is able to dominate over the increasing levels of gas motions and turbulence expected in the outer regions of clusters $^{21}$. Over the last $\sim 5$ Gyr Perseus has continued to grow as gas accretes onto it from the surrounding cosmic web in a highly asymmetric manner, yet this appears to have had no impact on the cold front structure. It also shows that many generations of rising bubbles from AGN feedback (which rise $\sim 20$ times faster than sloshing cold fronts and so should overtake or pass through them), have had no discernible impact, suggesting that rising bubbles can pass through cold fronts without affecting them.

If the hook interpretation is correct, the opening angle between the two edges is sensitive to the angle between the line-of-sight and the normal to the plane of the sloshing, $\theta$. The opening angle between the two edges increases as $\theta$ increases (supplementary figure 5). The observed opening angle in our Chandra observation is $\sim 30$ degrees, from which we infer that $\theta \leq 20$ degrees (i.e. the sloshing is mostly perpendicular to the line of sight, which is also in agreement with the observed spiral structure). Recently it has been found that if sloshing motions dominate the lineof-sight velocity gradient seen in the core with Hitomi ${ }^{26}$, the observed line widths and shifts are consistent with a significant inclination relative to the line-of-sight, potentially up to $\sim 45$ degrees 27 .

Since the sloshing needs to be mostly perpendicular to the line of sight to allow the hook to be seen, this may have prevented hook features from being observed in other cold front systems. Other factors which may also have facilitated the observation of such a feature in Perseus include the larger spatial scale of the hook in a large scale cold front (the edges are split by $\sim 100 \mathrm{kpc}$ ), combined with reduced projection effects away from the cluster core, and the large angular size of Perseus owing to its closeness. 


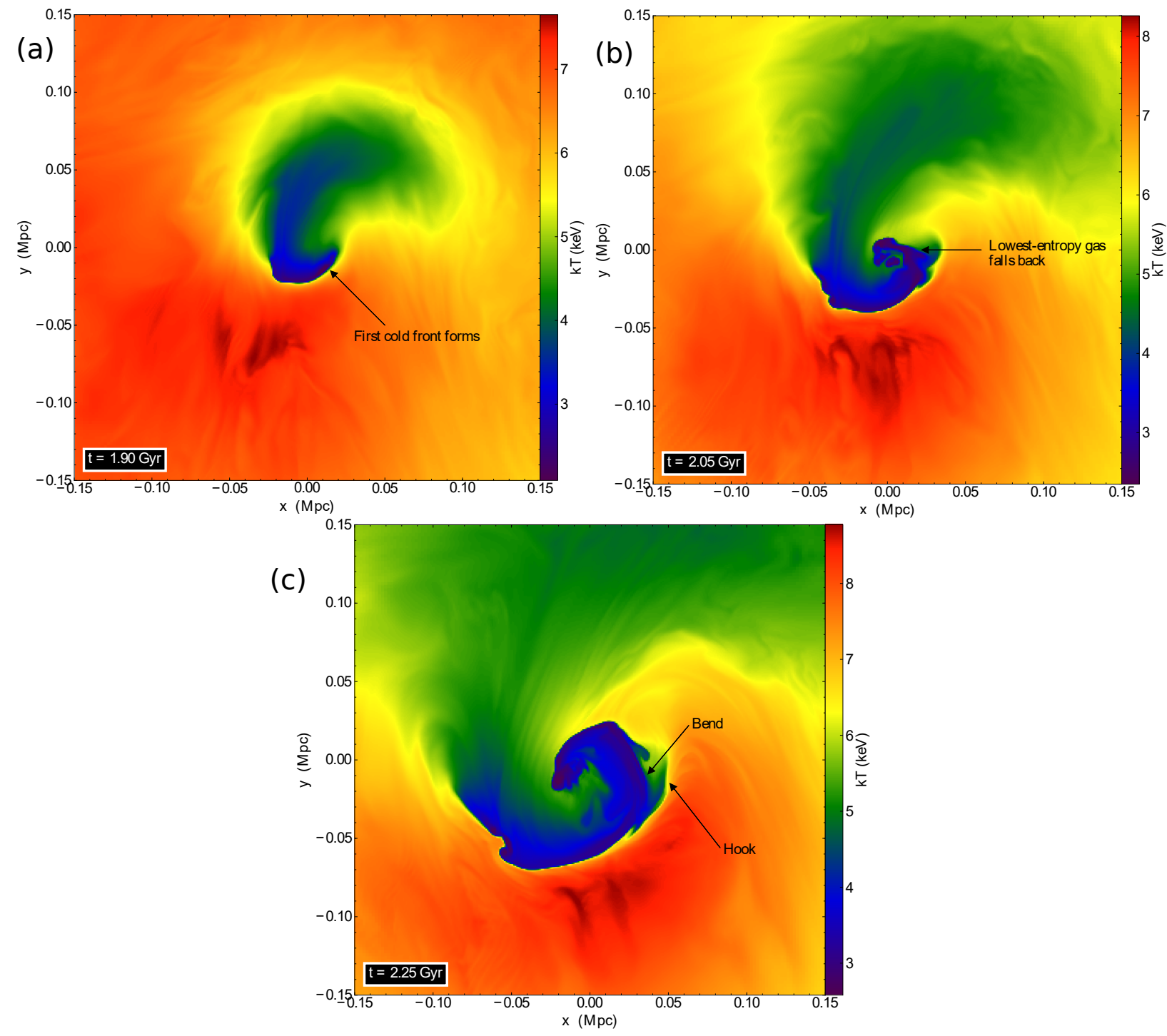

Figure 3 Simulations of cold front development with time. Simulated slices of the temperature maps for three time intervals showing the development of the hook structure as the cold front forms $s^{917}$, as described in the main text. The video of this simulation is available here (https://vimeo.com/236813999). The time stamp shows the time since the start of the simulation. The sloshing starts $1.3 \mathrm{Gyr}$ into the simulation, so the ages of the cold fronts shown in these panels are 0.6, 0.75 and 0.95 Gyr. The hook feature rises out with the cold front and survives out to the final epoch covered by the simulation, at which the cold front has risen to a radius of $450 \mathrm{kpc}$ (see supplementary figure 4). 


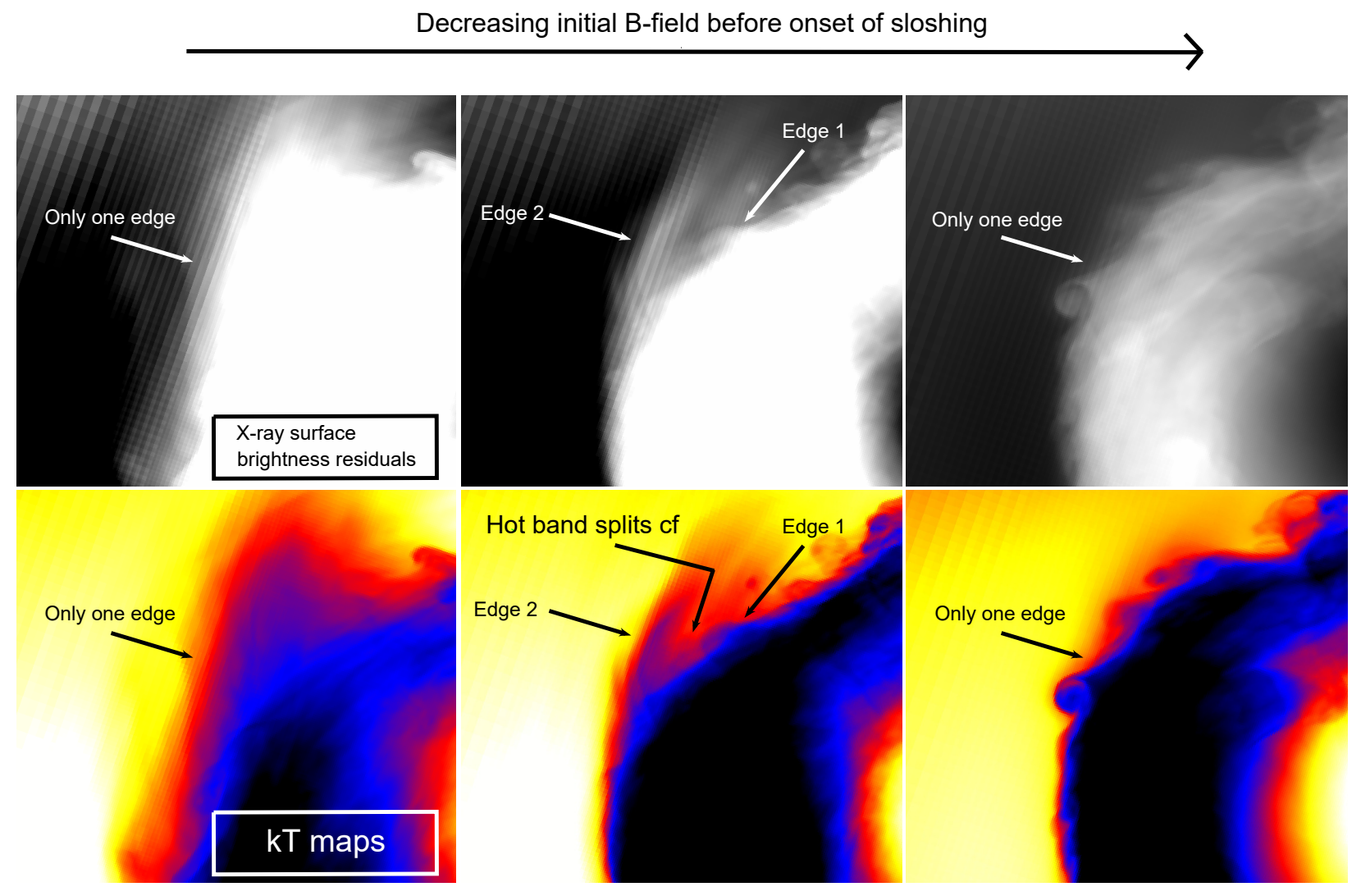

Figure 4 Simulations of cold front development with time. Comparing simulations ${ }^{9 \mid 17}$ of cold front development for decreasing initial magnetic field strength from left to right. The top row shows the $\mathrm{X}$-ray surface brightness residuals after dividing out the azimuthal average. The bottom row shows the temperature maps. In the left hand column, the initial magnetic field is strongest $(\beta=100)$ and prevents the cold front from splitting. In the middle column, the initial magnetic field is halved $(\beta=200)$, and is not strong enough to prevent the splitting, resulting in the same double edge feature that we observe. In the right hand column $(\beta=500)$, this even weaker magnetic field cannot form the split cold front, which instead forms $\mathrm{KHI}$ roll structures curving the other way. In these time slices of the simulation, the cold front is 2.7Gyr old, but the same behaviour with magnetic field is seen in all the epochs of the simulation.

\section{References}

1. Markevitch, M. \& Vikhlinin, A. Shocks and cold fronts in galaxy clusters. Physics Reports 443, 1-53 (2007). astro-ph/0701821.

2. Zuhone, J. A. \& Roediger, E. Cold fronts: probes of plasma astrophysics in galaxy clusters. Journal of Plasma Physics 82, 535820301 (2016). 1603.08882.

3. Simionescu, A. et al. Large-scale Motions in the Perseus Galaxy Cluster. ApJ 757, 182 (2012). 1208.2990. 
4. Markevitch, M., Ponman, T. J., Nulsen, P. E. J. \& Bautz, M. W. Chandra Observation of Abell 2142: Survival of Dense Subcluster Cores in a Merger. ApJ 541, 542-549 (2000). astro-ph/0001269.

5. Vikhlinin, A., Markevitch, M. \& Murray, S. S. A Moving Cold Front in the Intergalactic Medium of A3667. ApJ 551, 160-171 (2001). astro-ph/0008496.

6. Lyutikov, M. Magnetic draping of merging cores and radio bubbles in clusters of galaxies. MNRAS 373, 73-78 (2006). astro-ph/ 0604178.

7. Asai, N., Fukuda, N. \& Matsumoto, R. Three-dimensional Magnetohydrodynamic Simulations of Cold Fronts in Magnetically Turbulent ICM. ApJ 663, 816-823 (2007). astro-ph/ 0703536.

8. Dursi, L. J. \& Pfrommer, C. Draping of Cluster Magnetic Fields over Bullets and Bubbles Morphology and Dynamic Effects. ApJ 677, 993-1018 (2008). 0711.0213.

9. ZuHone, J. A., Markevitch, M. \& Lee, D. Sloshing of the Magnetized Cool Gas in the Cores of Galaxy Clusters. ApJ 743, 16 (2011). 1108.4427.

10. Rossetti, M. et al. Abell 2142 at large scales: An extreme case for sloshing? A\&A 556, A44 (2013). 1305.2420 .

11. Walker, S. A., Fabian, A. C. \& Sanders, J. S. Large-scale gas sloshing out to half the virial radius in the strongest cool core REXCESS galaxy cluster, RXJ2014.8-2430. MNRAS 441, L31-L35 (2014). 1402.6894.

12. ZuHone, J. A., Markevitch, M. \& Johnson, R. E. Stirring Up the Pot: Can Cooling Flows in Galaxy Clusters be Quenched by Gas Sloshing? ApJ 717, 908-928 (2010). 0912.0237.

13. Roediger, E. et al. Gas sloshing, cold fronts, Kelvin-Helmholtz instabilities and the merger history of the cluster of galaxies Abell 496. MNRAS 420, 3632-3648 (2012). 1112 . 1407.

14. Roediger, E., Kraft, R. P., Forman, W. R., Nulsen, P. E. J. \& Churazov, E. Kelvin-Helmholtz Instabilities at the Sloshing Cold Fronts in the Virgo Cluster as a Measure for the Effective Intracluster Medium Viscosity. ApJ 764, 60 (2013). 1211.4874.

15. Walker, S. A. et al. Is there a giant Kelvin-Helmholtz instability in the sloshing cold front of the Perseus cluster? MNRAS 468, 2506-2516 (2017). 1705.00011.

16. Werner, N. et al. Deep Chandra observation and numerical studies of the nearest cluster cold front in the sky. MNRAS 455, 846-858 (2016). 1506.06429 .

17. ZuHone, J. A., Kowalik, K., Öhman, E., Lau, E. \& Nagai, D. The Galaxy Cluster Merger Catalog: An Online Repository of Mock Observations from Simulated Galaxy Cluster Mergers. ApJS 234, 4 (2018). 
18. Fabian, A. C. Observational Evidence of Active Galactic Nuclei Feedback. ARA\&A 50, 455489 (2012). 1204.4114.

19. Fabian, A. C. et al. Do sound waves transport the AGN energy in the Perseus cluster? MNRAS 464, L1-L5 (2017). 1608.07088 .

20. Zhuravleva, I. et al. Turbulent heating in galaxy clusters brightest in X-rays. Nature 515, 85-87 (2014). 1410.6485 .

21. Lau, E. T., Kravtsov, A. V. \& Nagai, D. Residual Gas Motions in the Intracluster Medium and Bias in Hydrostatic Measurements of Mass Profiles of Clusters. ApJ 705, 1129-1138 (2009). 0903.4895 .

22. Sanders, J. S., Fabian, A. C., Russell, H. R., Walker, S. A. \& Blundell, K. M. Detecting edges in the X-ray surface brightness of galaxy clusters. MNRAS 460, 1898-1911 (2016). 1605.02911 .

23. Walker, S. A., Sanders, J. S. \& Fabian, A. C. Applications for edge detection techniques using Chandra and XMM-Newton data: galaxy clusters and beyond. MNRAS 461, 684-697 (2016). 1606.01255 .

24. Ascasibar, Y. \& Markevitch, M. The Origin of Cold Fronts in the Cores of Relaxed Galaxy Clusters. ApJ 650, 102-127 (2006). astro-ph/ 0603246.

25. Roediger, E. \& Zuhone, J. A. Fast simulations of gas sloshing and cold front formation. MNRAS 419, 1338-1349 (2012). 1109.2593.

26. Hitomi Collaboration \& et al. The quiescent intracluster medium in the core of the Perseus cluster. Nature 535, 117-121 (2016). 1607 .04487.

27. ZuHone, J. A., Miller, E. D., Bulbul, E. \& Zhuravleva, I. What Do the Hitomi Observations Tell Us About the Turbulent Velocities in the Perseus Cluster? Probing the Velocity Field with Mock Observations. ApJ 853, 180 (2018). 1708 . 07206.

28. Snowden, S. L., Mushotzky, R. F., Kuntz, K. D. \& Davis, D. S. A catalog of galaxy clusters observed by XMM-Newton. A\&A 478, 615-658 (2008). 0710.2241.

29. Wang, Q. D. \& Walker, S. X-ray mapping the outer regions of galaxy clusters at $\mathrm{z}=0.23$ and 0.45. ArXiv:1401.6205 (2014). 1401.6205.

30. Urban, O. et al. Azimuthally resolved X-ray spectroscopy to the edge of the Perseus Cluster. MNRAS 437, 3939-3961 (2014). 1307 .3592.

31. Moretti, A., Campana, S., Lazzati, D. \& Tagliaferri, G. The Resolved Fraction of the Cosmic X-Ray Background. ApJ 588, 696-703 (2003). arXiv:astro-ph/0301555. 
32. Walker, S. A., Fabian, A. C., Sanders, J. S., Simionescu, A. \& Tawara, Y. X-ray exploration of the outskirts of the nearby Centaurus cluster using Suzaku and Chandra. MNRAS 432, 554-569 (2013). 1303.4240.

33. Walker, S. A., Fabian, A. C. \& Sanders, J. S. An XMM-Newton view of the merging activity in the Centaurus cluster. MNRAS 435, 3221-3230 (2013). 1308 . 2090.

34. Smith, R. K., Brickhouse, N. S., Liedahl, D. A. \& Raymond, J. C. Collisional Plasma Models with APEC/APED: Emission-Line Diagnostics of Hydrogen-like and Helium-like Ions. ApJ 556, L91-L95 (2001). arXiv:astro-ph/0106478.

35. Kalberla, P. M. W. et al. The Leiden/Argentine/Bonn (LAB) Survey of Galactic HI. Final data release of the combined LDS and IAR surveys with improved stray-radiation corrections. A\&A 440, 775-782 (2005). arXiv:astro-ph/ 0504140.

36. Sanders, J. S. \& Fabian, A. C. A deeper X-ray study of the core of the Perseus galaxy cluster: the power of sound waves and the distribution of metals and cosmic rays. MNRAS 381, 13811399 (2007). 0705.2712 .

37. Russell, H. R., Sanders, J. S. \& Fabian, A. C. Direct X-ray spectral deprojection of galaxy clusters. MNRAS 390, 1207-1216 (2008). 0808 . 2371.

38. Sanders, J. S. et al. A very deep Chandra view of metals, sloshing and feedback in the Centaurus cluster of galaxies. MNRAS 457, 82-109 (2016). 1601.01489.

Correspondence Correspondence and requests for materials should be addressed to S.A.W. (stephen.a.walker@ nasa.gov).

Acknowledgements S.A.W. was supported by an appointment to the NASA Postdoctoral Program at the Goddard Space Flight Center, administered by the Universities Space Research Association through a contract with NASA. A.C.F. acknowledges support from ERC Advanced Grant FEEDBACK.

Competing interests The authors declare no competing financial interests.

Author contributions S.A.W. wrote the manuscript with comments from all the authors. S.A.W. performed the Chandra and XMM-Newton data analysis and lead the Chandra proposal. J.Z. produced the galaxy cluster sloshing simulations. 


\section{Methods}

Here we outline our analysis procedures. All errors quoted are at the $1 \sigma$ level unless otherwise specified.

Chandra image analysis. All the Chandra observations used are tabulated in supplementary table 1. For all Chandra analysis we used the latest version of CIAO (4.9). CHANDRA_REPRO was used to process the events files, while light curves in the $0.5-7.0 \mathrm{keV}$ band were assessed, with the routine LC_SIGMA_CLIP used to identify and remove and periods where the count rate deviated by more than $2 \sigma$ from the mean. Background images were obtained from stowed background, which were cleaned and processed in the same way as the observations, and projected into the same coordinate system. These backgrounds were rescaled to ensure they have the same high energy $(9.5-12.0 \mathrm{keV})$ count rates as the observations.

FLUX_OBS was used to obtain background subtracted images and exposure maps in seven narrow energy bands (1.2-1.5, 1.5-2.0, 2.0-2.5, 2.5-2.75, 2.75-3.0, 3.0-3.5, 3.5-4.0 keV), which were exposure corrected and then added, to produce the image shown in the left hand panel of Fig. 2. WAVDETECT was used to identify and remove point sources.

XMM image analysis. The XMM observations used to produce the $0.7-7.0 \mathrm{keV}$ image in the top panel of Fig. 1 are tabulated in supplementary table 2. We used only the MOS observations, following ${ }^{3}$. The data reduction and image processing were performed using the XMM-Newton Extended Source Analysis Software (XMM-ESAS), following the standard methodology 28 .

Spectral analysis. The Chandra spectral fitting and background modelling follows the procedures described in 29 . Stowed backgrounds, scaled to match the 9-12 keV count rate, were used to remove the particle background. To model the soft X-ray background, we used the best fitting background model from the Suzaku and ROSAT background fields ${ }^{30}$, which consists of three thermal components modelling the local hot bubble, the Galactic halo and a potential $0.6 \mathrm{keV}$ foreground component. We used a powerlaw of index 1.4 to model the cosmic X-ray background from unresolved point sources, and the normalization of this powerlaw was calculated using the model for the cumulative flux distribution of point sources 31 , using the threshold flux to which we can resolve point sources in our Chandra fields, following the methods outlined in 32 .

For the XMM spectral fitting and modeling, we follow the procedures from ${ }^{33}$. In addition to the background model, two gaussians were used to model the instrumental lines ( $\mathrm{Al} \mathrm{K} \alpha, 1.49$ $\mathrm{keV}$ amnd $\mathrm{Cu} \mathrm{K} \alpha, 1.75 \mathrm{keV}$ ), with their normalisations set as free parameters. Residual soft proton contamination was modelled as a broken powerlaw. The powerlaw normalisation for the cosmic X-ray background from unresolved point sources was calculated using the XMM-ESAS task POINT_SOURCE.

To test the robustness of the soft X-ray background modeling, we repeated each spectral fit 
using the range of background component values found in ${ }^{30}$ for the 8 different background Suzaku fields spaced equally around the cluster. Varying the background components by these ranges had negligible effect on the spectral fits to the intracluster medium.

In the spectral fits we fit the intracluster medium with an absorbed APEC ${ }^{34}$ model in XSPEC 12.9.1, using the extended C-statistic. The column density was fixed the $\mathrm{LAB}^{35}$ value of $1.45 \times 10^{21}$ $\mathrm{cm}^{-2}$, the metal abundance was fixed to 0.3 times solar metallicity, the redshift was fixed to $\mathrm{z}=0.01756^{26}$, and we fit for the temperature and density.

Temperature map. The temperature map shown in Fig. 2 was obtained by extracting spectra from a grid of partially overlapping ellipses, which are orientated to follow the broad surface brightness distribution. Each ellipse is shifted relative to the nearest one by half the length of its minor axis, so that each ellipse overlaps with at least 8 neighbouring regions, over which an average is found. The best fit temperature for each ellipse is found, and the temperature map is produced by averaging over the ellipses. This overlapping method acts to smooth the temperature map and reduce noise. Extracting regions from a fixed grid ensures that we avoid any artifacts from the binning process. Each ellipsoidal region contains at least 2000 counts after background subtraction.

The widescale temperature map shown in Fig. 1 was obtained from the shallow XMMmosaic using larger overlapping regions consisting of elliptical sectors spanning a radial range of 4 arcmins and with an azimuthal opening angle of 25 degrees, where the curvature of the ellipse is set to match the curvature of the cold front's X-ray surface brightness. Each sector was shifted relative to its neighbouring one by either a radial shift of 2 arcmins, or an azimuthal shift of 10 degrees. Again this overlapping method helps smooth the temperature map, reducing noise.

Temperature profiles. Temperature and surface brightness profiles over directions of interest are shown in supplementary figure 1. Profile 1 passes over the outermost edge. Profile 2 goes over the cold hook from one side to the other. Profile 3 goes over the hot band splitting the edges. Profile 4 goes over the innermost edge. The temperature profiles obtained in supplementary figure 1 were produced by extracting spectra in regions large enough to contain 2000 background subtracted counts, which were aligned with the surface brightness features. The regions were spaced 8 arcsecs apart, much smaller than the typical bin width of $\sim 0.5$ arcmin, to ensure that the profile shapes were not affected by our choice of where to place our bins. The spectral extraction regions are shown in supplementary figure 2. Deprojections were performed using the deprojection code DSDEPROJ ${ }^{3637}$.

Surface brightness profile fitting and edge width measurements. From the deprojected temperature and density profiles across the outer edge (edge 2 in Fig. 2 and profile 1 in supplementary figure 1), we find that the temperature jumps from $3.75 \pm 0.5 \mathrm{keV}$ to $7.0 \pm 1.0 \mathrm{keV}$, while the gas density falls from $5.2 \pm 0.2 \times 10^{-4} \mathrm{~cm}^{-3}$ to $3.7 \pm 0.3 \times 10^{-4} \mathrm{~cm}^{-3}$. The Coulomb mean free path given by 1 


$$
\lambda=15 \mathrm{kpc}(T / 7 \mathrm{keV})^{2}\left(n_{\mathrm{e}} / 10^{-3} \mathrm{~cm}^{-3}\right)^{-1}
$$

inside the edge is $\lambda_{\text {in }}=8.0 \pm 2.1 \mathrm{kpc}(20.7 \pm 5.6 \mathrm{arcsec})$, and outside is $\lambda_{\text {out }}=40.5 \pm 12.0 \mathrm{kpc}(105 \pm 31$ arcsec). Therefore the mean free path for diffusion from the inner (brighter) side to the outer side, given by,

$$
\lambda_{\text {in } \rightarrow \text { out }}=\lambda_{\text {out }} \quad T_{\text {in }} / T_{\text {out }} \quad G(1) / G\left(\left(T_{\text {in }} / T_{\text {out }}\right)^{1 / 2}\right)
$$

where $G(x)=\left[\operatorname{erf}(x)-x \operatorname{erf}^{\prime}(x)\right] / 2 x^{2}$ (where $\operatorname{erf}(x)$ is the error function) is $\lambda_{\text {in } \rightarrow \text { out }}=23.2 \pm 6 \mathrm{kpc}$ $(60.0 \pm 15 \mathrm{arcsec})$. From fitting the surface brightness jump with a broken powerlaw smoothed by a Gaussian ${ }^{16[38}$, the observed width of the edge is consistent with zero, with a conservative ( $\left.3 \sigma\right)$ upper limit on the width of 8 arcsecs $(3.1 \mathrm{kpc}$ ), which is 3.1/23.2=0.13 \pm 0.04 times smaller than $\lambda_{\text {in } \rightarrow \text { out }}$. The reduced $\chi^{2}$ for the best fit is 1.05 for 36 degrees of freedom. The density jump ratio is a factor of $0.7 \pm 0.06$.

The pressure inside the outer edge is $2.0 \pm 0.4 \times 10^{-3} \mathrm{keV} \mathrm{cm}^{-3}$, and outside is $2.3 \pm 0.5 \times 10^{-3}$ $\mathrm{keV} \mathrm{cm}{ }^{-3}$, so the pressure is consistent with being continuous. This is not inconsistent with the potential presence of a magnetic draping layer, since it would probably be very thin ${ }^{6 / 9}$, and cannot be detected with the sensitivity available in our observations.

For the inner edge (edge 1 in Fig. 2, profile 4 in supplementary figure 1), the temperature jumps from $3.5 \pm 0.3 \mathrm{keV}$ to $5.0 \pm 0.3 \mathrm{keV}$, while the density drops from $7.0 \pm 0.2 \times 10^{-4} \mathrm{~cm}^{-3}$ to $5.7 \pm 0.2 \times 10^{-4} \mathrm{~cm}^{-3}$, which yields $\lambda_{\text {in }}=5.3 \pm 1 \mathrm{kpc}(14 \pm 2.4 \mathrm{arcsec}), \lambda_{\text {out }}=13.4 \pm 1.7 \mathrm{kpc}(35 \pm 4.4$ arcsec), and $\lambda_{\text {in } \rightarrow \text { out }}=9.6 \pm 1.5 \mathrm{kpc}(25 \pm 4 \mathrm{arcsec})$. Again the $3 \sigma$ upper limit on the width of the edge is 8 arcsecs $(3.1 \mathrm{kpc})$, which is $3.1 / 9.6=0.3 \pm 0.05$ times smaller than $\lambda_{\mathrm{in} \rightarrow \mathrm{out}}$. The reduced $\chi^{2}$ for the best fit is 1.06 for 43 degrees of freedom. The density jump ratio is a factor of $0.8 \pm 0.05$.

With the depth of data available, we are able to identify any surface brightness jumps corresponding to density changes greater than 5 percent with at least $5 \sigma$ signficance, so any density jumps we do not identify must be less than this 5 percent level.

Details of the simulations. The simulations we used are based on those from 9 , but with an improved treatment of gravity, and improved spatial resolution ${ }^{13}$. In these simulations, sloshing is initiated in a massive cool core cluster similar to Perseus $\left(\mathrm{M}_{200}=10^{15}\right.$ solar mass) by an infalling smaller subcluster (one fifth the mass of the main cluster), moving on a trajectory that gives it an impact parameter of $500 \mathrm{kpc}$, and starting from a distance of $3 \mathrm{Mpc}$. The temperature, gas density and mass profiles of the main cluster were originally designed to emulate those observed in the similarly massive cool core cluster Abell 2029, and have been found to provide a reasonable approximation to the Perseus cluster ${ }^{27}$. These simulations use the rigid potential approximation ${ }^{13}$, in which rather than using the N-body method to follow the evolution of the dark matter haloes of the clusters directly, a rigid potential is assumed for both clusters. The simulations are run in the rest 
frame of the most massive cluster and corrections are applied to account for the fact that the rest frame of the main cluster is not an inertial frame.

Data availability The data that support the plots within this paper and other findings of this study are available from the corresponding author upon reasonable request.

\section{Supplementary figures}


Supplementary figure 1 | Profiles of temperature and surface brightness across the cold front edges. The top 2 panels show the Chandra image and temperature map. In the bottom 4 panels we show the temperature and surface brightness profiles along 4 directions shown by the lines labelled in the images. The blue curves are the best Gaussian smoothed broken powerlaw fits to the two main surface brightness edges.
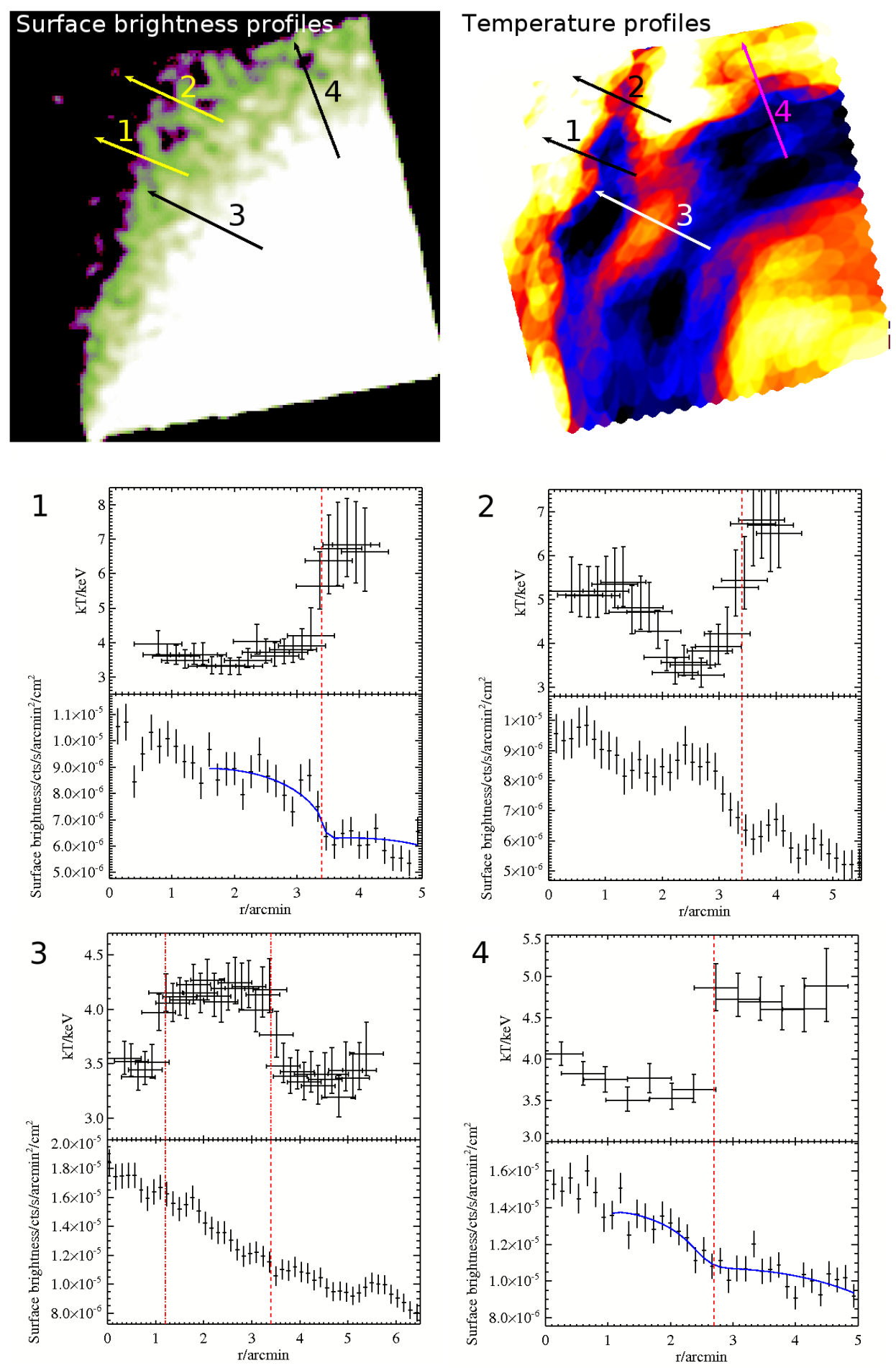


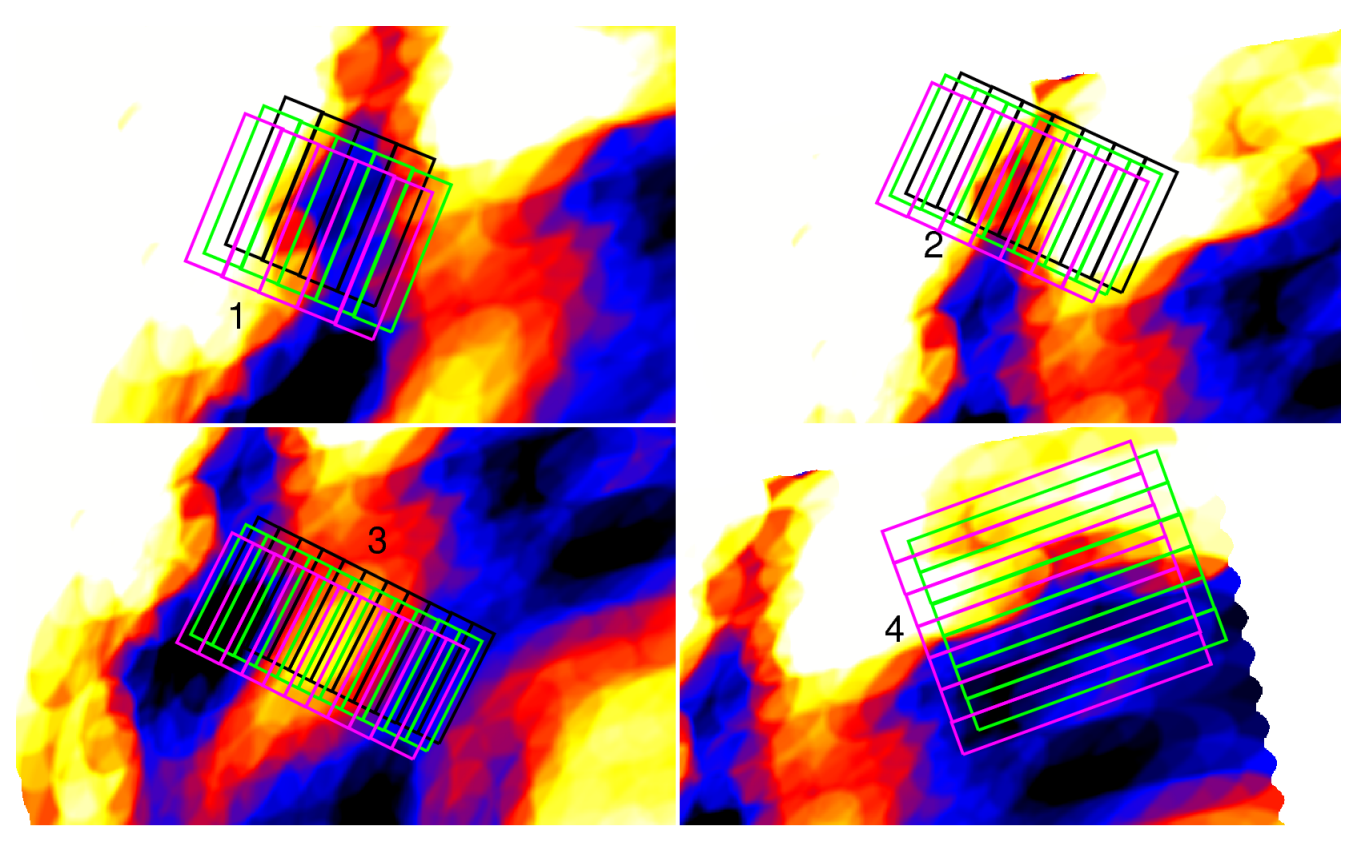

Supplementary figure 2 | Spectral extraction regions for the temperature profiles. The rectangular boxes show the spectral extraction regions for the four temperature profiles shown in supplementary figure 1, which are overlaid on the temperature map. Deprojected temperature profiles were obtained in each coloured strip (pink, green, black), each of which is offset parallel to the profile direction by around 8 arcsec to ensure that our profiles are not affected by the positions of the regions we use. The offsets perpendicular to the profile direction are included just to make it easier to see the different regions in this figure. 


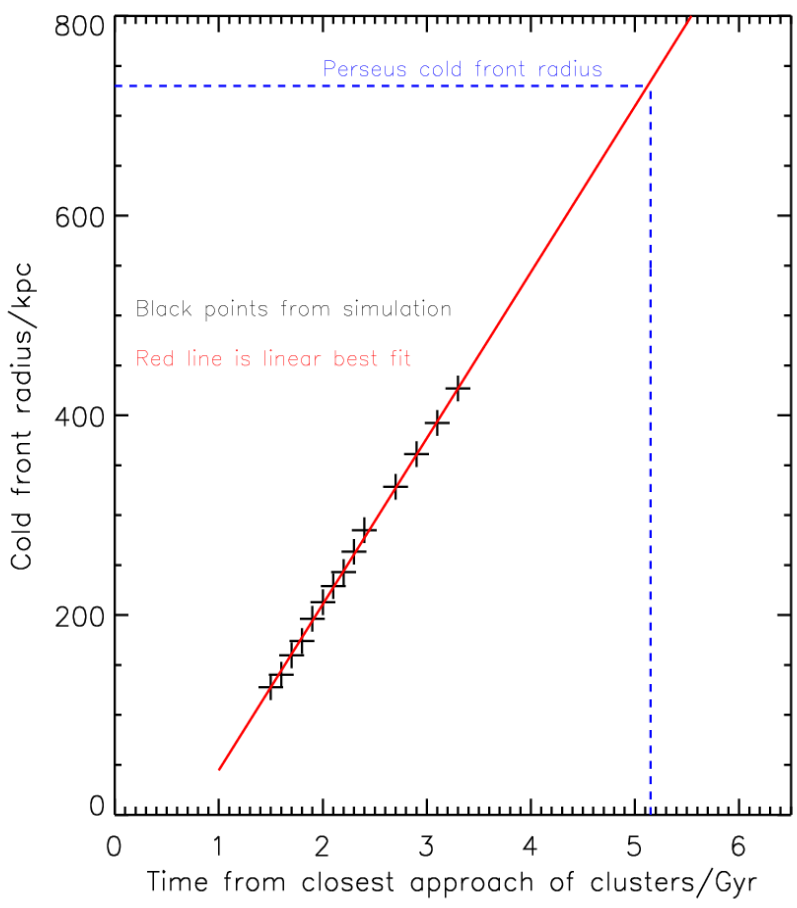

Supplementary figure 3 | The time evolution of the cold front radius. The black points show the radius of the outer cold front versus the time from the closest approach of the clusters, from the simulations, which extend out to 3.3Gyr from the moment of closest approach. The relation is very well fit with a linear relation, with the cold front rising at constant speed (as has been observed in earlier works ${ }^{13}$ ). The best fit linear relation is shown as the red line. Extending this linear best fit out to the radius of the observed cold front in Perseus (730kpc), gives an estimated age of 5.2 Gyr. From the outermost cold front radius covered by the simulations (450kpc) to the Perseus cold front radius $(730 \mathrm{kpc})$, the thermodynamic and mass profiles of the undisturbed cluster follow the same basic powerlaws that they follow in the region covered by the simulations shown here $(150-450 \mathrm{kpc})$, so there is no reason for the speed on the cold front to change from 450 to $730 \mathrm{kpc}$. Conservatively, we quote as estimate of $~ 5 \mathrm{Gyr}$ for the age of the cold front. 


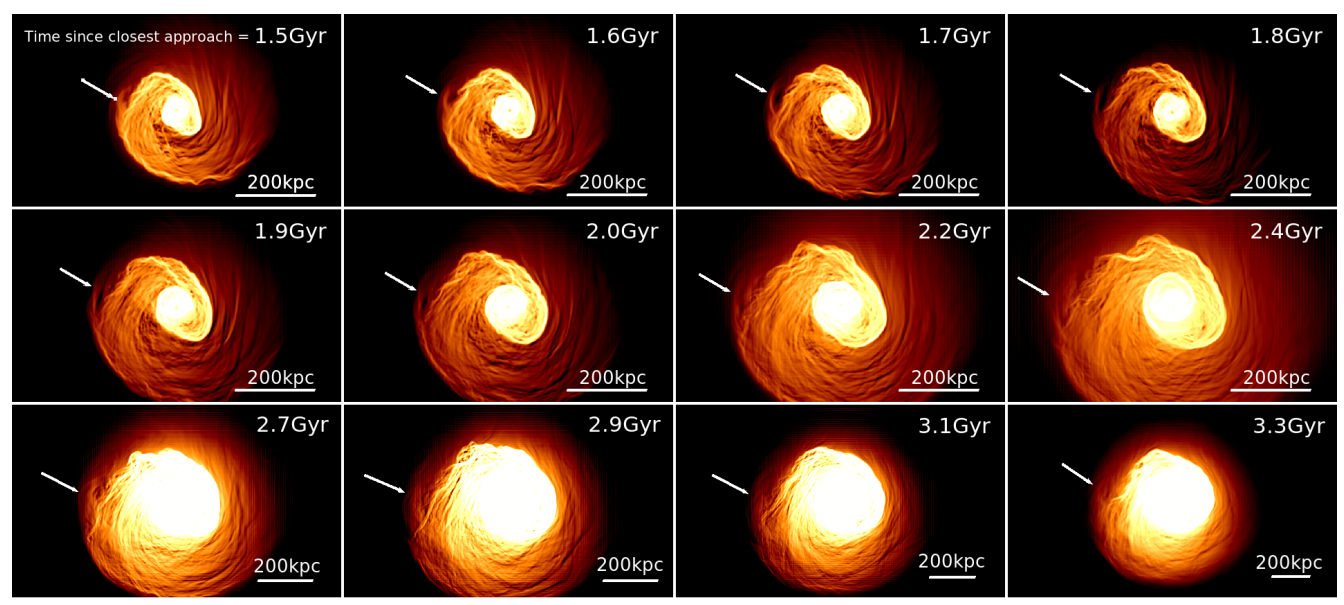

Supplementary figure 4 | The time evolution of the hook. Time slices of the simulation (filtered by the GGM filter) showing that the hook feature (highlighted by the white arrow) continues to survive into the latest epoch covered by the simulation as the cold front rises outwards. From $t=1.5 \mathrm{Gyr}$, to the final epoch at $\mathrm{t}=3.3 \mathrm{Gyr}$, the cold front rises from $120 \mathrm{kpc}$ to $450 \mathrm{kpc}$ (nearly a factor of 4 increase in radius), with the hook feature surviving and rising out with the cold front. In the later time slices we zoom out in order to show the full sloshing activity. 

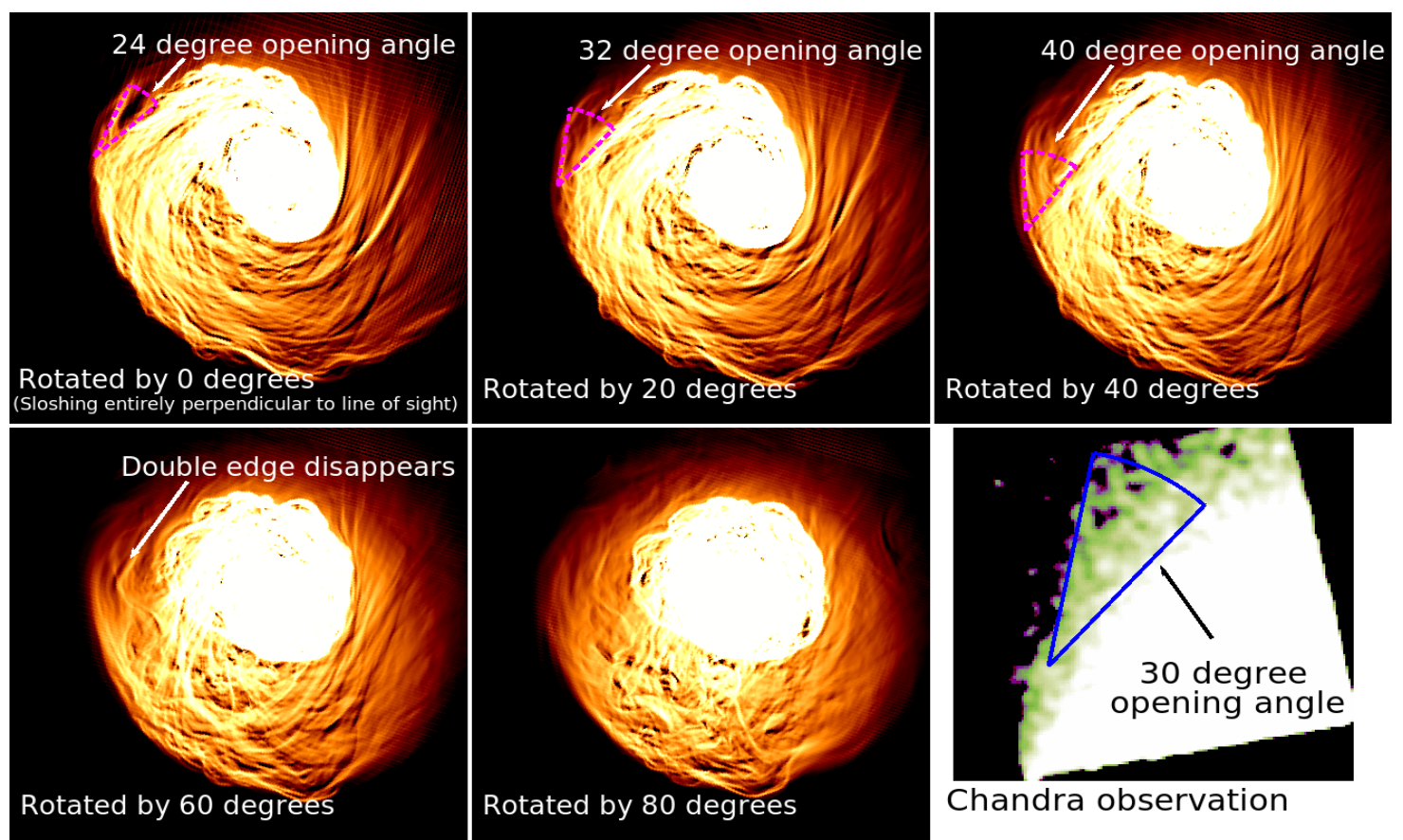

Supplementary figure 5 | Comparing the double edge structure to rotated simulations. Here we show the effect of rotating the simulations about an axis perpendicular to the line of sight in the first 5 panels. The simulated X-ray emissivity has been filtered with a Gaussian Gradient Magnitude (GGM) filter to enhance the contrast of the edges. In the first panel (top left), the sloshing is entirely perpendicular to the line of sight, so the angle between the line of sight and the normal to the plane of the sloshing $(\theta)$ is zero. As the angle between the line of sight and the normal to the sloshing plane ( $\theta$ ) increases (i.e. as the sloshing occurs more along the line of sight), the opening angle between the two edges (shown by the magenta triangles) increases. When $\theta=60$ degrees, the double edge feature is no longer visible. In the sixth panel we show the observed opening angle between the edges in the Chandra data of 30 degrees (blue triangle), suggesting that the angle between the line of sight and the normal to the sloshing plane is at most 20 degrees. In this time slice, the cold front is $2.7 \mathrm{Gyr}$ old. The same behaviour is found for all of the other time slices out to the end of the simulation. In this time slice this distance from the core to the outer cold front is 350kpc. 
Supplementary table 1 | New Chandra observations of eastern cold front

\begin{tabular}{llll}
\hline Obs ID & RA & Dec & Exposure/ks \\
\hline 19565 & 03h 21m 59.00s & +41d 41' 31.10" & 42 \\
19938 & 03h 21m 59.00s & +41d 41' 31.10" & 51
\end{tabular}

Supplementary table 2 | XMM-Newton observations for mosaic

\begin{tabular}{|c|c|c|c|}
\hline Obs ID & $\mathrm{RA}$ & Dec & Exposure/ks \\
\hline 0085110101 & 03h 19m 48.16s & +41d 30' 42.1" & 52 \\
\hline 0085590201 & 03h 19m 49.69s & +41d 05' 47.0" & 40 \\
\hline 0151560101 & 03h 16m 42.99s & +41d 19' 29.0" & 23 \\
\hline 0204720101 & 03h 21m 38.59s & +41d 31' 43.0" & 13 \\
\hline 0204720201 & 03h 23m 23.60s & +41d 31' 41.0" & 22 \\
\hline 0305690101 & 03h 18m 02.69s & +41d 16' 60.0" & 25 \\
\hline 0305690301 & 03h 19m 50.59s & +41d 53' 34.3" & 18 \\
\hline 0305690401 & 03h 21m 53.70s & +41d 49' 30.1" & 26 \\
\hline 0305720101 & 03h 17m 57.99s & +41d 45' 57.0" & 11 \\
\hline 0305720301 & 03h 22m 15.99s & +41d 11' 28.0" & 17 \\
\hline 0405410101 & 03h 21m 04.26s & +41d 56' 05.0" & 16 \\
\hline 0405410201 & 03h 18m 39.93s & +41d 06' 31.4" & 16 \\
\hline 0673020201 & 03h 23m 07.63s & +41d 12' 35.6" & 23 \\
\hline 0673020301 & $03 \mathrm{~h} 22 \mathrm{~m} \mathrm{10.00s}$ & +41d 23' 00.0" & 14 \\
\hline 0673020401 & 03h 21m 14.16s & +41d 10' 52.3" & 17 \\
\hline
\end{tabular}

